\title{
Solar radioastronomy with the LOFAR (LOw Frequency ARray) radio telescope
}

\author{
Stephen M. White ${ }^{a}$, Namir E. Kassim ${ }^{b}$ and William C. Erickson ${ }^{b}$ \\ ${ }^{a}$ Dept. of Astronomy, Univ. of Maryland, College Park MD 20742, USA \\ ${ }^{b}$ Remote Sensing Div., Naval Research Lab., Washington D.C., USA
}

\begin{abstract}
The Low Frequency Array (LOFAR) will be a radio astronomy interferometric array operating in the approximate frequency range 10-240 MHz. It will have a large collecting area achieved using active dipole techniques, and will have maximum baselines of up to $500 \mathrm{~km}$ to attain excellent spatial resolution at long wavelengths. The Sun will always be in LOFAR's field of view during daylight hours, and particularly during periods of high solar activity the Sun will be a prominent (and highly variable) feature of the low-frequency sky. A diverse range of low-frequency emissions is generated by the Sun that carry information about processes taking place in the Sun's atmosphere. Study of these emissions with LOFAR will make possible major advances in our understanding of particle acceleration and shocks in the solar atmosphere, and of coronal mass ejections and their impact on the Earth. In this paper we summarize LOFAR's capabilities and discuss the solar science that LOFAR will address.
\end{abstract}

Keywords: Low-frequency radio astronomy, Solar radio astronomy, Solar activity, Coherent emission processes

\section{INTRODUCTION}

Following the discovery of cosmic radio signals at long wavelengths by Karl Jansky, the early development of radio astronomy continued at low frequencies, for the obvious reason that low-frequency technology was then easier to implement. The earliest radio telescopes all operated at low frequencies, and this is where the first major scientific discoveries of radio astronomy took place; radio astronomy only migrated to the higher microwave frequencies once the technology made it feasible. Microwave frequencies have two crucial advantages over longer wavelengths: for a given array dimension the spatial resolution is better; and microwave frequencies do not suffer from the ionospheric effects (perturbations of the ray paths from a straight line due to inhomogeneities in the ionosphere's electron plasma content, and hence refractive index) that plague low frequencies on long baselines. For this reason, low-frequency radio astronomy, at least in the US, languished in the 1980s once the Clark Lake Radio Observatory ${ }^{1}$ (operated by the University of Maryland) was closed. Elsewhere, low-frequency astronomy continues to be pursued at exiting facilities such as Nançay ${ }^{2,3}$ (France), Gaurabidanur $^{4}$ and Ooty $^{5}$ (India), and UTR- $2^{6}$ (Ukraine), and in newer projects such as the Giant Meterwave Radio Telescope ${ }^{7,8}$ (GMRT) in India.

However, since low-frequency radio astronomy is relatively cheap, astronomers in the US realized that the Very Large Array (VLA) could be outfitted with simple dipole receivers at negligible cost: this led to the addition of first $327 \mathrm{MHz}$ and then $74 \mathrm{MHz}^{9}$ systems on each VLA antenna. Over the years, experience was gained with these systems and it was realized that a combination of deconvolution and self-calibration techniques was capable of removing the effects of the ionosphere and achieving nearly instrument-limited image quality even at high spatial resolution. ${ }^{10-12}$ This makes it attractive to reconsider the prospects for high spatial resolution imaging at meter wavelengths and longer, and a suggestion for a lowfrequency array exploiting these developments ${ }^{13}$ together with the recognition that a number of important astrophysical topics can best be tackled in this frequency range (e.g., the epoch of reionization) has led to the LOw Frequency ARray (LOFAR) project.

Further author information: (Send correspondence to $\mathrm{S}$. White)

SMW: E-mail: white@astro.umd.edu

NEK: E-mail: kassim@ rsd.nrl.navy.mil, Address: Remote Sensing Division, Naval Research Laboratory, Code 7213, Washington D.C. 20375-5351, USA

WCE: E-mail: bill.erickson@utas.edu.au, Address: as per NEK 
LOFAR will use active dipole receiving elements with an intrinsically large field of view, sensitive at the lower frequencies to essentially the whole sky at once. This means that during the day the Sun will always be in the LOFAR field of view, and its activity must be taken into account, both as a fluctuating source in the field and as a source of ionospheric inhomogeneity. Solar research will necessarily form a major component of LOFAR studies, and interest in LOFAR coincides with the realization that Space Weather, which can be studied at long wavelengths, is playing an increasingly important role in our technologically-dominated society. The purpose of this paper is to summarize LOFAR and to discuss its value for solar and Space Weather studies.

\section{THE LOW FREQUENCY ARRAY}

LOFAR is presently conceived to be a joint project of ASTRON (the Netherlands Foundation for Research in Astronomy, based in Dwingeloo, The Netherlands), the Naval Research Laboratory, and MIT/Haystack Observatory. Here we summarize the main elements of the LOFAR project, using the current information presented at the LOFAR web site, http: //www. lofar.org.

\subsection{The Proposed Telescope}

LOFAR will operate as an interferometric array consisting of a large number of dipoles grouped into 100 "stations" each consisting of $100-1000$ dipoles spread over an area $\sim 100 \mathrm{~m}$ in diameter. The large number of dipoles is necessary to achieve sufficient effective collecting area in order to reach the desired sensitivity. The dipoles within each station will be phased together to form small beams, avoiding the problem of correlating the output from every dipole with every other dipole (the number of cross-correlations goes as $\mathrm{N}^{2}$, so dividing $\mathrm{N}$ dipoles into $n \sim \mathrm{N} / 100$ stations and only having to correlate the combined outputs from the $n$ stations simplifies the correlation problem enormously). At least two types of receiving elements are expected to be needed: one set operating from $15-90 \mathrm{MHz}$ (this is such a wide range that one element may not be able to cover it), and another from 110 - $240 \mathrm{MHz}$. The frequency break between these receiving elements can naturally be made to coincide with the band used for FM radio stations ( $88-108 \mathrm{MHz})$, which is full of strong interfering signals throughout the world.

At each station, a "beam" pointing in a certain direction will be formed by combining the signals from all dipoles after inserting a position-dependent delay in each signal that has the effect of adding the flux that arrives from one direction on the sky and canceling out the flux from other directions. The dipoles themselves have a very wide field of view: this process of forming a beam from dipoles spread out over a whole station produces a beam whose size is determined by the size of the station (i.e., the beam pattern has a size similar to that of a single dish with an aperture equal to the size of the station). Since the beams are formed electronically by delaying signals without mechanically changing the direction the dipoles point, one can split the signals from the dipoles and form several different beams illuminating different directions on the sky (within the reception pattern of a single dipole) simultaneously, without loss of sensitivity; the limit to this process is provided by the need to analyze the data from each beam.

The current design for LOFAR features the following elements:

- Over $10^{4}$ dual polarization dipoles optimized for operation at 10-90 MHz.

- Over $2 \times 10^{5}$ dual polarization dipoles arranged in a $4 \times 4$ compound element array and optimized for operation at 110-220 MHz.

- Between 2 and 8 independent beams that will allow simultaneous observing programs to be executed.

- Maximum baselines of up to $500 \mathrm{~km}$.

- All dipoles distributed over 60 to 165 stations (the number of dipoles per station varies inversely with the number of stations).

- At least $25 \%$ of the total collecting area to be located within a $2 \mathrm{~km}$ diameter "virtual core" where the full signal from each antenna is transported to a central processing site. These signals can be phased up to form a sensitive 2 km diameter "super-station".

- Rapid frequency switching (of order $1 \mathrm{msec}$ ) 
- An instantaneous RF bandwidth of at least $32 \mathrm{MHz}$ (potentially more if faster commercial digitizers become available in the near future) will be digitized at the antenna level. (All simultaneous beams will have to use the common bandwidth.)

- Beamforming for the virtual core together with all correlation and pipeline data processing will be carried out at a central processing site.

The virtual core provides high brightness sensitivity for low surface brightness extended objects, while the outlier stations provide high spatial resolution that avoids the confusion limitation prevalent in low-resolution low-frequency radio astronomy. At present a log-spiral design is favored for the arrangement of stations outside the virtual core. Bandwidth synthesis will be exploited to fill in the $u, v$ plane. Approximate sensitivities of the full array are given in Table 1. The sensitivities quoted are given for each beam, a single polarization, an integration time of $1 \mathrm{~s}$ and a $4 \mathrm{MHz}$ bandwidth. Resolutions are based on a Virtual Core diameter of $2 \mathrm{~km}$ and a maximum baseline of $400 \mathrm{~km}$.

Table 1. Basic design parameters of LOFAR $(\mathrm{VC}=$ Virtual Core, Array $=$ full array $)$.

\begin{tabular}{lcccccccc}
\hline $\begin{array}{l}\text { Frequency } \\
(\mathrm{MHz})\end{array}$ & Effective Area & Tsys & \multicolumn{2}{c}{ Sensitivity (mJy) } & \multicolumn{2}{c}{ Resolution } & \multicolumn{2}{c}{ Beamsize } \\
& $\left(\mathrm{m}^{2}\right)$ & $(\mathrm{K})$ & Array & VC & Array (") & VC (") & Array (') & VC (deg) \\
\hline 15 & $1.3 \times 10^{6}$ & 131,000 & 98 & 400 & 12 & 41 & 1260 & 90 \\
30 & $3.3 \times 10^{5}$ & 23,000 & 68 & 280 & 6.2 & 21 & 650 & 90 \\
75 & $5.2 \times 10^{4}$ & 2450 & 46 & 190 & 2.5 & 8.3 & 250 & 90 \\
120 & $3.3 \times 10^{5}$ & 820 & 2.4 & 10 & 1.5 & 5.2 & 160 & 23 \\
200 & $1.2 \times 10^{5}$ & 270 & 2.2 & 9 & 0.9 & 3.1 & 95 & 23 \\
\hline
\end{tabular}

With baselines of up to $500 \mathrm{~km}$ required and the need for a low-interference environment, the siting of LOFAR will not be a trivial exercise. Presently, sites in the Netherlands, in the south-west of the US (spread across New Mexico and Texas, but not co-located with the VLA), and Western Australia (in the Murchison region) are being investigated.

\subsection{Imaging with LOFAR}

The imaging problem faced by LOFAR is a formidable one. At a spatial resolution of order arcseconds and a wide field of view, there can easily be $10^{10}$ resolution elements and $10^{7}$ sources in a single image. LOFAR, in common with all interferometers, measures spatial Fourier components of the sky brightness distribution and these Fourier components must be inverted in order to recover the sky brighness distribution. The field of view of LOFAR is so large that the conventional imaging assumption that neglects the curvature of the sky cannot be made: the three-dimensional nature of the inversion problem must be addressed. At present this is done by dividing the three-dimensional field of view into many small two-dimensional "facets". ${ }^{14}$ This is numerically expensive, and LOFAR is relying on continued advances in computing speed to make this feasible. The computational driver for extending the polyhedron algorithm to lower frequencies is the number of facets that are required to divide up the surface of the field of view $\left(N_{\text {facet }} \propto \lambda B / D^{2}\right.$, where $B$ is the baseline length, $\lambda$ is the wavelength and $D$ is the aperture size of individual receiving elements). This can exceed $10^{4}$ for LOFAR.

In addition, since the dimensions of the array are comparable to the height of the ionosphere, a single station looking at different parts of the field of view will see different isoplanatic patches and suffer different effects. In effect, the antennabased phase correction needed to account for the ionosphere will vary across the field of view of a single station, unlike the case at higher frequencies where the field of view is smaller than the isoplanatic patch on the sky and a single phase correction applies everywhere within the primary beam of a given antenna. In effect, this means that self-calibration must solve for a time-varying position-dependent phase correction for each antenna; another way to think of this is that LOFAR needs to derive a global ionospheric model over all the visible sky on a time-dependent basis in order to determine the corrections needed to remove the ionosphere and achieve instrument-limited imaging. 
One aspect of dealing with this problem is to develop a model of the low-frequency sky that can be used to initiate the self-calibration cycle. The sky model will consist of at least the brighter known sources throughout the sky, mapped as a function of position and frequency, that provides an initial model for calibration and that allows a first-order determination of position-dependent ionospheric phase corrections. The sky model will have to be developed iteratively, with each new observation adding information to the model. As a by-product of the removal of the ionospheric phase noise from the data using many different antennas and many different directions on the sky, the ionosphere can be characterized in detail, providing a boon for ionospheric science.

Adding to these difficulties is the problem of interference, prevalent in the frequency range of LOFAR. This will be addressed by a combination of filtering out the more stable sources, adaptive nulling of sources using electronic beam shaping, real-time identification and rejection, and self-calibration techniques that can recognize the properties of interference and excise it.

\subsection{Primary Science Goals}

The goal of LOFAR is to build a low-frequency array with excellent sensitivity and spatial resolution that can attack a range of problems that are really only accessible at low radio frequencies. We mention several highlights here; a more complete description may be found at the web site http://www.lofar.org/science/urd100/science_case.html.

- The epoch of reionization (EOR): After recombination of the elements at a redshift of 1100 , the baryonic matter in the Universe remained neutral until the first stars and galaxies started to emit ionizing radiation, at which point the intergalactic medium was reionized. At least three different types of sources have been suggested as contributing to the radiation that was responsible for reionization: emission from the first generation of stars; radiation released in the collapse of the first galaxy-sized halos; and emission from an early generation of luminous quasars. The EOR can be detected at low frequencies because it caused a small step in the temperature of the background radiation. ${ }^{15}$ The predicted spectroscopic signature is generated on large scales $\left(10^{\prime}\right)$ at the rest frequency of the neutral hydrogen line $(1420 \mathrm{MHz})$, but redshifted to LOFAR frequencies by the expansion of the Universe. Alternatively, the neutral intergalactic medium may be detected in absorption towards the first quasars to form. ${ }^{16}$ The exact frequency at which the temperature step is detected, or the redshift of the absorption, yields the time of reionization which is an important parameter in the cosmological history of the universe.

- High-redshift radio galaxies: these are the oldest and most massive distant objects, providing important constraints upon early star formation epochs. The most efficient method to date for finding very high redshift radio galaxies uses the correlation between radio spectrum steepness and redshift. At their rest frequency synchrotron losses generate a nonthermal continuum spectrum which steepens above $1 \mathrm{GHz}$. For distant objects this "break frequency" is shifted to longer and longer wavelengths in the observer's frame, making them steep spectrum sources which are ideal for detection by a low frequency radio telescope such as LOFAR.

- Mapping galactic cosmic rays: LOFAR will play an important role by mapping the space distribution and energy spectrum of cosmic-ray electrons. Optically thick thermal sources at known distances, such as HII regions, are observed in absorption; a technique that can be exploited only at low frequencies and yields directly line-of-sight cosmic-ray synchrotron emissivities. Comparisons with observations at other wavelengths, including the distributed Galactic gamma-ray emission, will provide important tools for decoupling the matter, cosmic-ray, and magnetic field distributions in the Galaxy.

- Surveys of the bursting and transient universe: Any transient phenomena that accelerate particles to nonthermal energies will tend to produce radio emission with a steep spectrum that makes it most easily detectable at low radio frequencies. This is the reason why pulsars were first discovered at low frequencies. LOFAR's large instantaneous field of view means that LOFAR can effectively monitor a large fraction of the sky at all times, averaging on many different time scales. This makes possible for the first time a sensitive unbiased survey for astronomical transients, and the nature of electronic pointing will allow rapid response to transients.

The solar-related science goals of LOFAR will be discussed in the following sections. We note that LOFAR nicely complements another radio telescope project, the Frequency Agile Solar Radiotelescope (FASR), whose frequency range will begin at the upper limit of LOFAR's range (probably with some overlap) and extend to above $20 \mathrm{GHz}$. FASR is a solar-dedicated telescope carrying out imaging spectroscopy of the radio Sun. ${ }^{17}$ 


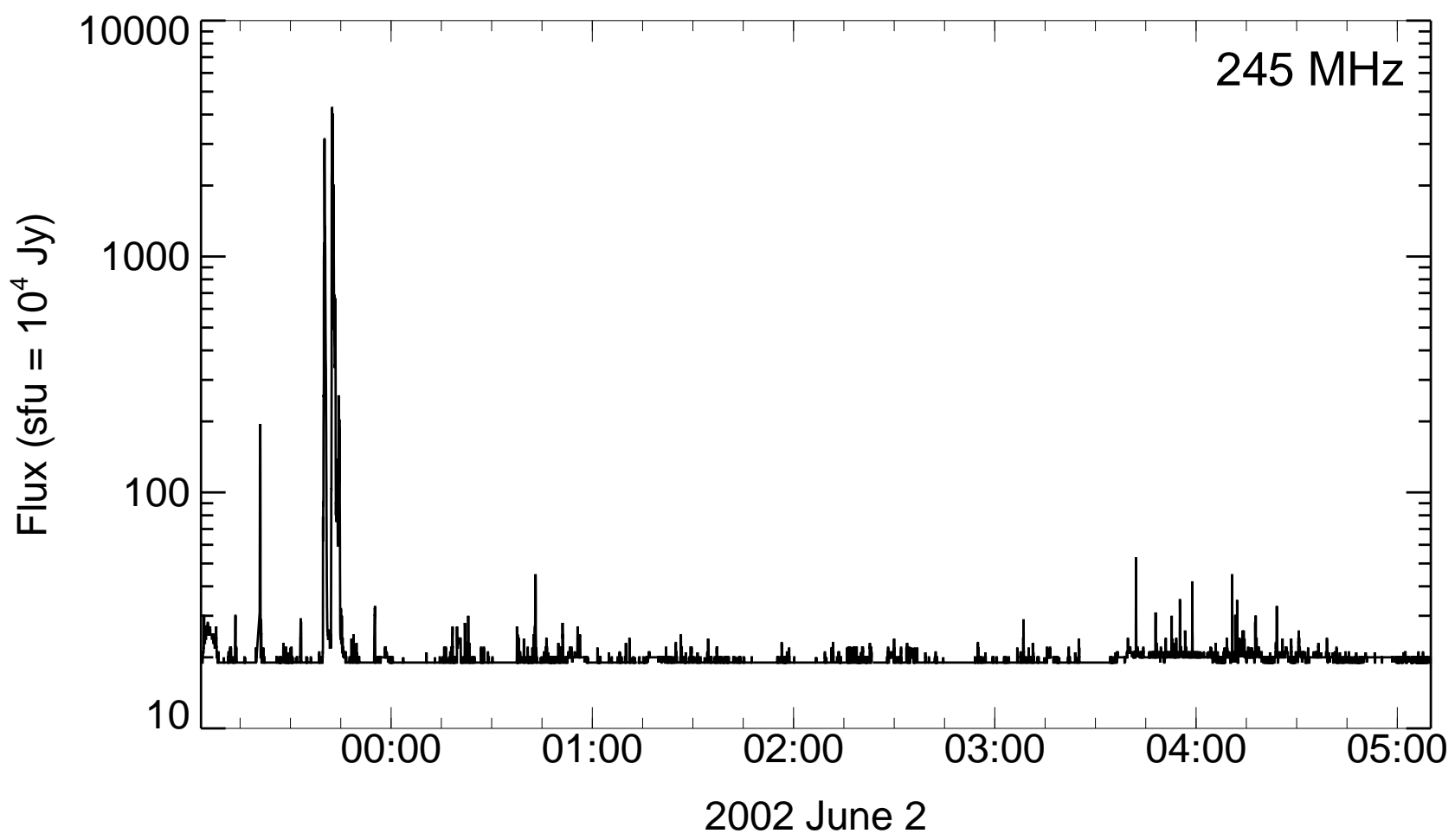

Figure 1. Variability of the solar low-frequency flux on a day of activity. These data are $245 \mathrm{MHz}$ data at $1 \mathrm{~s}$ time resolution from the Learmonth IPS/US Air Force patrol telescope. A sequence of flares occurs during this period; the brightest radio emission comes from a relatively small flare (GOES class C5) that occurs at around 23:45 UT on June 1.

\section{THE SUN AT LOW FREQUENCIES}

The flux from the quiet Sun is not as spectacular at low frequencies as it is at high frequencies: the Sun's atmosphere is optically thick due to thermal free-free emission from the corona at a height substantially above the photosphere, and the quiet-Sun flux can be estimated by assuming a brightness temperature of $10^{6} \mathrm{~K}$ over a radius of 1 to $2 \mathrm{R}_{\odot}$, depending on frequency, leading to roughly $20000(f / 100 \mathrm{MHz})^{2} \mathrm{Jy}\left(1\right.$ solar flux unit $\left.=1 \mathrm{sfu}=10^{4} \mathrm{Jy}\right)$. The quiet-Sun flux does however vary considerably with the solar cycle; it has been reported to be as low as $2000 \mathrm{Jy}$ at $50 \mathrm{MHz}$ during solar minimum, ${ }^{18}$ which is much fainter than sources such as Cas A and Cygnus A. The Sun moves $2.5^{\prime \prime}$ relative to the background sky per minute; since that is the resolution of LOFAR at the higher frequencies, solar motion will have to be taken account of in calibrating and mapping daytime observations. Furthermore, the solar flux can vary dramatically at low frequencies: an example is shown in Figure 1, where the quiet-Sun flux is just $19 \mathrm{sfu}$, but the peak flux during flares rises to 300 times this level, when (at $\sim 10^{8} \mathrm{Jy}$ ) the Sun will be easily the brightest object in the sky.

Figure 2 shows the frequency dependence of the bright event in Fig. 1 as a dynamic spectrum. This event shows two common types of low-frequency solar radio emission ${ }^{19-21}$ : "Type III" bursts, which drift very rapidly from high to low frequencies and are known to be due to beams of fast electrons travelling away from the solar surface on open magnetic field lines; and "Type II" bursts, which drift much more slowly and frequently appear as two distinct bands of emission. Both these bursts are believed to emit via the plasma radiation mechanism, in which electrostatic Langmuir waves are excited at the local electron plasma frequency, $f_{p}=9000 \sqrt{n_{e}}$ with $n_{e}$ being the electron density $\left(\mathrm{cm}^{-3}\right)$, and then converted to propagating electromagnetic modes in the plasma at the fundamental $f_{p}$ and the harmonic $2 f_{p}$ frequencies. The frequency of the radio emission therefore can be translated directly into an electron density, and the frequency drift rate can be converted into a speed if the density gradient in the corona is known. For Type II bursts this procedure usually results in a speed of order $500-1000 \mathrm{~km} \mathrm{~s}^{-1}$, while the Alfvén speed at the emitting height is though to be typically several hundred $\mathrm{km} \mathrm{s}^{-1}$ : this led to the idea that Type II bursts are driven by shocks travelling at several times the local Alfvén speed. 


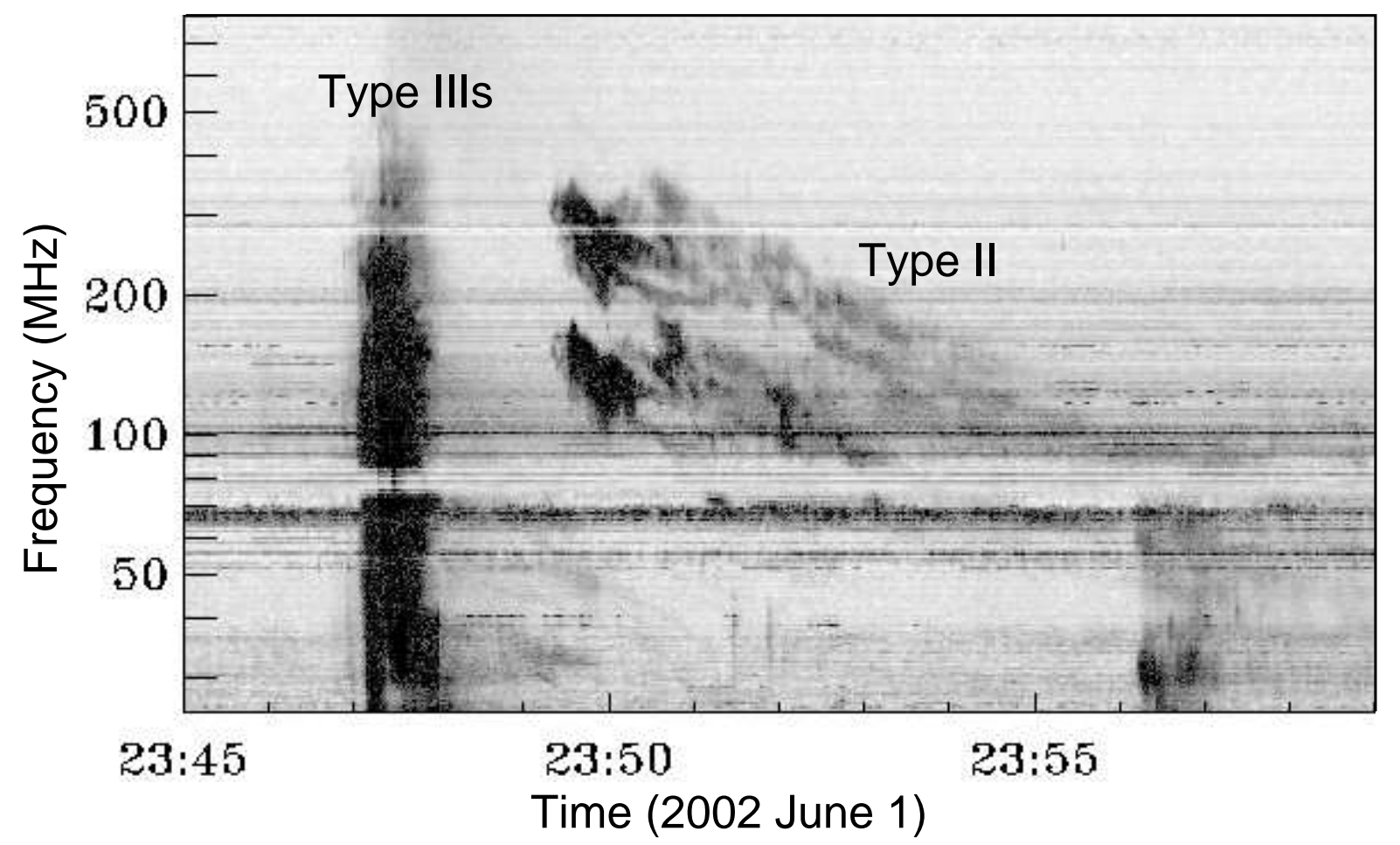

Figure 2. Dynamic spectrum (plot of frequency versus time, with frequency decreasing from top to bottom of the plot) of the event at 23:45 on 2002 June 1 responsible for the brightest fluctuations in Fig. 1. The data are from the Hiraiso spectrograph in Japan. This event is a classic Type III/Type II event: the impulsive onset of the flare is signalled by the group of fast-frequency-drift (Type III) bursts at 23:47 UT, indicating electrons travelling outwards from the Sun on open field lines at speeds of order $0.2 \mathrm{c}$. They are followed by a much more slowly drifting pair of bands (Type II), thought to be fundamental and harmonic plasma emission from a shock moving at $\sim 600 \mathrm{~km} \mathrm{~s}^{-1}$.

Type II bursts always follow flares, and hence the associated shocks must somehow be flare-related; there remains a controversy as to whether they are in fact driven primarily by coronal mass ejections ${ }^{22}$ (CMEs) that occur with flares. Type III bursts need not be flare associated, although they assuredly indicate episodes of acceleration of electrons to nonthermal energies, and further indicate that those electrons have access to open field lines. The location of the acceleration sites in the corona is difficult to identify: the highest frequency of (fundamental) emission displayed by a burst need not correspond to the density in the acceleration region because free-free absorption in the ambient thermal plasma increases dramatically as density increases, and can prevent us from seeing the electron beam low in the corona. In the case of Type II bursts, the Alfvén speed is higher in the low corona, and a shock may not form in front of a disturbance originating at high densities until it reaches a height where its speed greatly exceeds the local Alfvén speed: this leads to Type II bursts having the appearance of just "turning on" several minutes after the flare onset, even though the disturbance driving the shock was probably launched at flare onset.

Another important burst type, not present in Fig. 2, is the Type IV burst. This is identified on dynamic spectra as a broadband continuum typically following the Type II in time. Such continua with similar appearances on dynamic spectra may well have quite different properties otherwise, and for this reason they have been difficult to study. ${ }^{23,24}$ Some of these continua are produced by sources that move out to great heights above the corona ("moving Type IVs" 25 ) and may well be associated with coronal mass ejections. However, none of these conjectured associations have been established, and this will be an important issue for LOFAR to address, as discussed in the next section. Two problems in particular have plagued such studies in the past: the apparent positions of radio sources in the solar corona can be affected by ducting, i.e., the positions often appear to be higher than they really are because flux emitted in a region close to the local plasma frequency is easily refracted along ducts of low plasma density, emerging from the ducts only when the frequency of the 


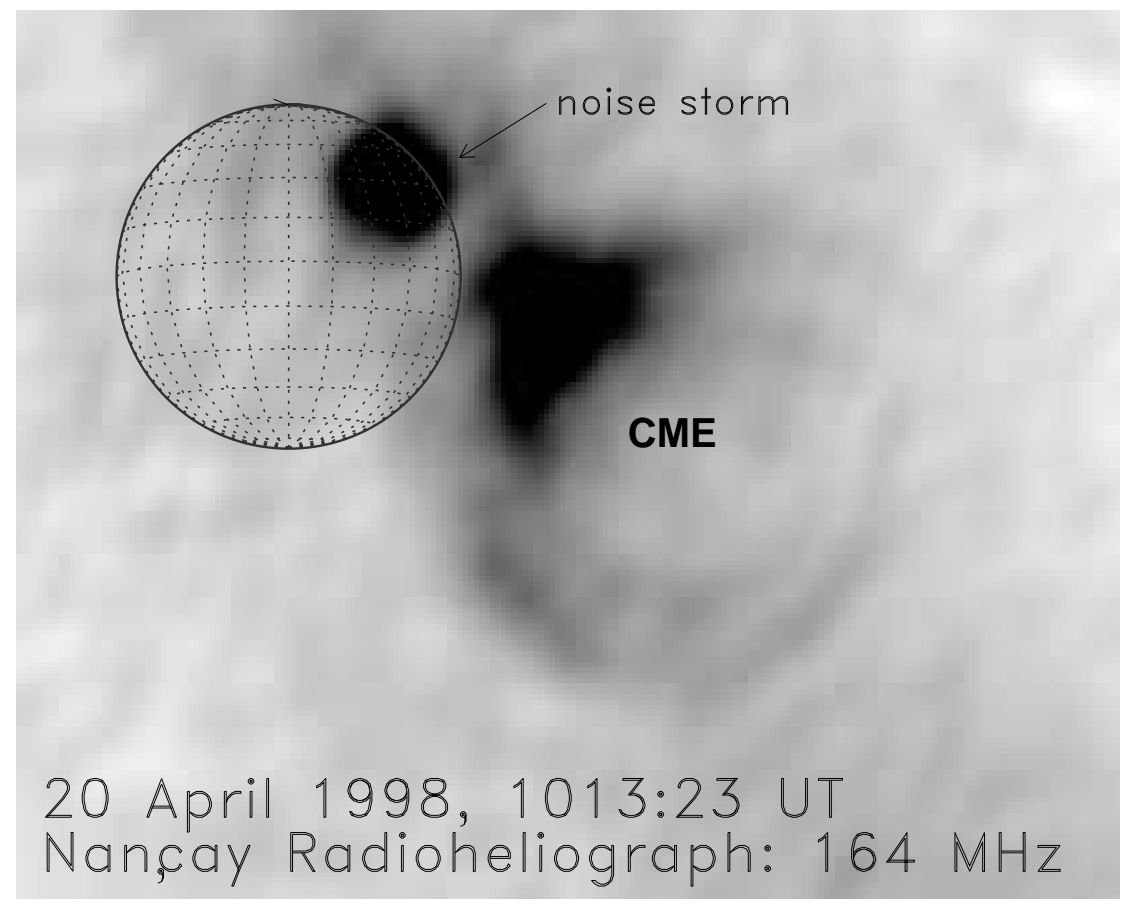

Figure 3. Direct detection of radio emission from a coronal mass ejection at $164 \mathrm{MHz} .^{29}$ The image is made with data from the Nançay radioheliograph. The flare occurred behind the solar limb so that the bright emission from low in the solar atmosphere is occulted. The image shows a noise storm (bright compact continuum source, unrelated to the flare) together with extended emission that outlines a coronal mass ejection observed simultaneously with the SOHO/LASCO coronagraph. The radio emisison from the CME is a broadband continuum with a falling spectrum, suggesting synchrotron radiation from electrons accelerated at the leading edge of the CME.

emission is well above the surrounding plasma frequency ${ }^{26}$ (in the absence of this effect sources would actually appear lower than they really are due to the radial decrease in plasma density in the solar wind ${ }^{27}$ ); and scattering intrinsic to the solar corona makes sources appear bigger than they truly are. ${ }^{28}$ Since both these effects are local to the Sun, they will be apparent to LOFAR as well; however, LOFAR's continuous frequency coverage will allow us to investigate position shifts and source sizes as a function of frequency in a way not possible with previous instruments and thus yield a much clearer picture of the sources of the radio emission. One problem that previous low-frequency telescopes have suffered that should not be a problem for LOFAR is errors in positions due to ionospheric refraction, since the ionosphere will be well characterised in the normal course of LOFAR analysis.

\section{SPACE WEATHER}

The field of Space Weather has taken on increasing importance in recent years, as the realization of just how damaging space influences on the terrestrial environment can be. Geomagnetic fluctuations caused by currents in the Earth's magnetosphere can overwhelm long-distance power grids and cause huge economic disruption, and threaten life if it causes power loss in communities that depend on electrical heating to survive the polar winters. Energetic particles accelerated close to the Sun can damage solid-state electronics on satellites, and there are numerous examples of satellite loss attributed to this cause; further, solar-induced heating of the ionosphere can cause it to expand upwards into the altitudes occupied by low-Earth-orbit satellites, increasing the drag on them and causing them to drop out of orbit and burn up in the Earth's atmosphere. Energetic particles also pose a health risk to astronauts and to high-altitude flyers such as commercial and military pilots, while ionospheric disturbances cause disruption to long-range radio communications.

As can be seen from this list, the sources of Space Weather are diverse. Coronal mass ejections play a major role in causing geomagnetic storms and disrupting the auroral particle belts, and any means of studying CMEs is of potential 
importance. LOFAR can play a role here because the plasma frequency associated with CMEs in the low corona, where they first become visible to coronagraphs, falls into LOFAR's frequency range and observable effects are to be expected. As noted above, some people believe that Type II radio bursts are driven by CMEs; moving Type IV bursts are more widely accepted as being associated with CMEs, but they are much rarer than Type IIs and few have been studied in recent years because no telescope has been available to image them (they typically occur well below Nançay's lower frequency of $164 \mathrm{MHz}$, but will fall squarely in LOFAR's frequency range). With its superior resolution and sensitivity and the ability to use imaging at closely spaced frequencies to sort out the effects of wave propagation in the solar corona, LOFAR will play a major role in settling these controversies.

There have been recent developments that encourage us to be confident that LOFAR will indeed be able to fulfil its promise of imaging CMEs. There are now a number of Nançay observations at $169 \mathrm{MHz}$ of emissions that are apparently moving with CMEs. Maia et. al (2000) report such emissions in several events associated with partly-occulted flares. The Nançay observations show unpolarized drifting features consistent with Type II emission, but too weak to be identified as such in spectrograph records. Bastian et. al (2001) ${ }^{29}$ analyzed one of these events further and showed that the moving feature reported by Maia et al. (2000) in fact lies at the base of the CME, but sensitive images show that there is also fainter emission outlining essentially the entire outer edge of the CME (Figure 3). From the broadband spectrum of this emission Bastian et al. (2001) suggested that in fact the emission mechanism is synchrotron emission from electrons accelerated at the leading edge of the CME rather than plasma emission as in a Type II burst. This emission directly outlines the $\mathrm{CME}$ and if it can be observed routinely, it will provide a more reliable means for detecting CMEs particularly in the low corona. The reason that it is observed in this particular event appears to be because the corresponding flare occurred over the limb and bright emissions at $164 \mathrm{MHz}$ arising from processes low in the corona were obscured from view. Normally such bright emissions dominate the available dynamic range of the images, making the lower surface brightness extended emission from the CME undetectable. LOFAR's characteristics will specifically help to avert this problem: the inner core of dipoles will provide excellent sensitivity to low surface brightness emission, and it should be capable of far superior dynamic range in its images thanks to the collecting area, the $u, v$ coverage and particularly the removal of ionospheric effects. The CME emission visible in Fig. 3 is too weak to be seen on dynamic spectra such as Fig. 2, so on current evidence it is possible that it is present in most CMEs but usually undetectable for the reasons given above, in which case LOFAR will detect a large fraction of all CMEs directly through such emission. Whether it can be detected from CMEs originating on the solar disk and travelling directly towards us is presently unknown, and probably cannot be answered until LOFAR begins operation.

Other aspects of Space Weather may also prove to be best addressed through low-frequency observations. The energetic protons and $\alpha$ particles that bombard the Earth following some solar events are an important feature of Space Weather and there are efforts to correlate their occurrence with phenomena in the solar corona. Cane et al. ${ }^{31}$ argue that essentially all solar energetic particle events are preceded by groups of Type III events that are long-lasting and occur only at low frequencies. They tend to occur after the Type II emission (if present) has started, and are always associated with CMEs. Since these long-lasting Type III bursts (labelled "Type III-l") do not need a Type II to be present and start at frequencies corresponding to densities much higher than are characteristic of the CME at the corresponding time, it is proposed that they are accelerated at heights below the leading edge of the CME. These Type III- $l$ bursts will be present in LOFAR's frequency range and if indeed they are tracers of solar energetic particle events, they will provide another important role for LOFAR in Space Weather.

\section{SOLAR RADAR EXPERIMENTS}

In addition to the passive detection of radio emission from CMEs in the solar corona, active detection of CMEs using radar techniques has great potential for Space Weather research. Solar radar with a suitably equipped transmitting facility and using LOFAR as the detecting and imaging element may provide a unique technique for detecting and tracking CMEs, opening up an entirely new and exciting field of solar research.

The interest in this proposal is generated by the results of a unique experiment carried out in the 1960's with the El Campo radar, built by the Lincoln Laboratory, which detected $38 \mathrm{MHz}$ radar echoes from the Sun for a period of 9 years. ${ }^{32}$

Huge, rapidly-moving targets were occasionally observed but this was before the space-borne coronagraph discovery of coronal mass ejections (CMEs), and the physical nature of these "targets" was a mystery. It is now thought that CMEs 
were being observed. Subsequent radar experiments have been unsuccessful, but none have had the combination of transmitting power and receiving area possessed by El Campo.

Radar experiments transmit a very narrow band signal and detect any frequency shifts in the reflections (or echoes). The Doppler shift introduced by different parts of an outward-moving CME will result in a characteristic frequency- and time-dependent signature in the reflected signal. The rich information inherent in this measurement could open an entirely new window on CME studies, yielding their angular distribution, ranges, and line-of-sight velocities. Combining the radial velocity obtained from the Doppler shift with the transverse velocity obtained from imaging would yield the CME total velocity vector. This may allow for accurate predictions of CME Earth-arrival times. Aside from the macroscopic physics of direct interest to the space weather program, there is great potential in unraveling the microscopic physics of the solar radar scattering mechanism. An understanding of this mechanism is key to solving the puzzles of the spectral shape, the large Doppler spread, the Doppler shift, and the variation in solar radar cross section observed by James. Various mechanisms have been suggested, including turbulence in the local medium, fluctuations in the altitude of the plasma resonance level due to electron density fluctuations in the solar wind, ion acoustic waves, and coherent lower hybrid waves. If the radar technique is successful, it will also provide a sensitive probe of turbulence in the solar corona.

Two transmitters for solar radar applications are being discussed at present. A monostatic $26 \mathrm{MHz}$ solar radar system has been proposed for Arecibo. ${ }^{33}$ The stand-alone version of this system will be powerful enough to confirm James' results using modern technology, with the advantage of the availability of the extensive monitoring of the upper solar atmosphere and CMEs at other wavelengths that James did not have at his disposal. This system would provide a steppingstone to a more powerful system that would use Arecibo as the transmitter and LOFAR as a receiving element to provide two-dimensional information. In addition, there is a Swedish proposal ${ }^{34}$ to construct a phased-array transmitting antenna ("LOIS"). The elements of the transmitter will be distributed over a large area so that the power from each element will be low enough to avoid creating plasma irregularities in the ionosphere, with the signals being phased to merge at the target location in the interplanetary medium. LOFAR would provide much greater collecting area, and hence sensitivity, than LOIS itself would achieve as the receiving element. If they are successful, these solar radar techniques could revolutionize the way in which we study coronal mass ejections.

\section{ACKNOWLEDGMENTS}

Research for this work at the University of Maryland was supported by NSF grants ATM 99-90809 and AST 01-38317, and NASA grants NAG 5-8192 and NAG 5-10175. Basic research in radio astronomy at the Naval Research Laboratory is supported by the Office of Naval Research. We thank Pat Crane for comments.

\section{REFERENCES}

1. W. C. Erickson, M. J. Mahoney, and K. Erb, “The Clark Lake Teepee-Tee telescope,” Astrophys. J. Supp. 50, pp. 403419, 1982.

2. Y. Avignon, J. Bonmartin, A. Bouteille, B. Clavelier, and E. Hulot, “The mark IV Nancay Radioheliograph,” Solar Phys. 120, pp. 193-204, 1989.

3. A. Kerdraon and J. Delouis, "The Nançay Radioheliograph," in Coronal Physics from Radio and Space Observations, G. Trottet, ed., p. 192, Springer, (Berlin), 1997.

4. R. Ramesh, K. Subramanian, and C. Sastry, "The gauribidanur radioheliograph.," Solar Phys. 181, p. 439, 1998.

5. S. Sukumar, T. Velusamy, A. Pramesh Rao, G. Swarup, and D. S. Bagri, "Ooty synthesis radio telescope - Design and performance," Bulletin of the Astronomical Society of India 16, pp. 93-110, 1988.

6. E. P. Abranin, L. L. Bazelian, N. I. Goncharov, V. V. Zaitsev, V. A. Zinichev, V. O. Rapoport, and I. G. Tsybko, "Positions of solar storm burst sources by observations with a heliograph based on the UTR-2 antenna at $25 \mathrm{MHz}$," Solar Phys. 66, pp. 393-409, 1980.

7. G. Swarup, "Giant metrewave radio telescope (GMRT) - Scientific objectives and design aspects," Indian Journal of Radio and Space Physics 19, pp. 493-505, 1990.

8. V. K. Kapahi and S. Ananthakrishnan, "Astronomy with the Giant Metrewave Radio Telescope (GMRT)," Bulletin of the Astronomical Society of India 23, p. 265, 1995.

9. N. E. Kassim, R. A. Perley, W. C. Erickson, and K. S. Dwarakanath, "Subarcminute resolution imaging of radio sources at $74 \mathrm{MHz}$ with the Very Large Array," AJ 106, pp. 2218-2228, 1993. 
10. T. N. LaRosa, N. E. Kassim, T. J. W. Lazio, and S. D. Hyman, "A Wide-Field 90 Centimeter VLA Image of the Galactic Center Region," AJ 119, pp. 207-240, 2000.

11. N. E. Kassim, T. E. Clarke, T. A. Enßlin, A. S. Cohen, and D. M. Neumann, "Low-Frequency VLA Observations of Abell 754: Evidence for a Cluster Radio Halo and Possible Radio Relics," Astrophys. J. 559, pp. 785-790, 2001.

12. C. K. Lacey, T. J. W. Lazio, N. E. Kassim, N. Duric, D. S. Briggs, and K. K. Dyer, "Spatially Resolved Thermal Continuum Absorption against Supernova Remnant W49B," Astrophys. J. 559, pp. 954-962, 2001.

13. N. E. Kassim and W. C. Erickson, "Meter- and decameter-wavelength array for astrophysics and solar radar," in Proc. SPIE Vol. 3357, p. 740-754, Advanced Technology MMW, Radio, and Terahertz Telescopes, Thomas G. Phillips; Ed., 3357, pp. 740-754, 1998.

14. T. J. Cornwell and R. A. Perley, "Radio-interferometric imaging of very large fields - The problem of non-coplanar arrays," Astron. Astrophys. 261, pp. 353-364, 1992.

15. N. Y. Gnedin and J. P. Ostriker, "Reionization of the universe and the early production of metals," ApJ 486, pp. 581$598,1997$.

16. C. Carilli, N. Y. Gnedin, and F. Owen, "Hi $21 \mathrm{~cm}$ absorption beyond the epoch of reionization," ApJ, 2002. in press.

17. T. S. Bastian, "The frequency agile solar radiotelescope," in SPIE Proceedings: Innovative Telescopes and Instrumentation for Solar Astrophysics, S. L. Keil and S. V. Avakyan, eds., SPIE, 2002. in press.

18. G. Thejappa and M. R. Kundu, "Unusually low coronal radio emission at the solar minimum," Solar Phys. 140, pp. 19-39, 1992.

19. J. P. Wild, S. F. Smerd, and A. A. Weiss, "Solar Bursts," Ann. Rev. Astron. Astrophys. 1, p. 291, 1963.

20. M. R. Kundu, Solar Radio Astronomy, Interscience Publishers, New York, 1965.

21. D. J. McLean, "Metrewave solar radio bursts," in Solar Radiophysics: Studies of Emission from the Sun at Metre Wavelengths, pp. 37-52, 1985.

22. E. W. Cliver, D. F. Webb, and R. A. Howard, “On the origin of solar metric type II bursts," Solar Phys. 187, pp. 89114, 1999.

23. R. D. Robinson, "Observations and interpretation of moving type iv solar radio bursts," Solar Phys. 60, p. 383, 1978.

24. R. D. Robinson, "A study of solar flare continuum events observed at metre wavelengths," Australian Journal of Physics 31, pp. 533-545, 1978.

25. R. T. Stewart, "Moving type iv bursts," in Solar Radiophysics, D. J. McLean and N. R. Labrum, eds., pp. 361-383, Cambridge University Press, (Cambridge), 1985.

26. R. A. Duncan, "Wave ducting of solar metre-wave radio emission as an explanation of fundamental/harmonic source coincidence and other anomalies," Solar Phys. 63, p. 389, 1979.

27. D. B. Melrose and G. A. Dulk, "Implications of liouville's theorem for the brightness temperatures of solar radio bursts," Solar Phys. 116, p. 141, 1988.

28. T. Bastian, "Angular scattering of solar radio emission by coronal turbulence," Astrophys. J. 426, p. 774, 1994.

29. T. S. Bastian, M. Pick, A. Kerdraon, D. Maia, and A. Vourlidas, "The coronal mass ejection of 1998 april 20: Direct imaging at radio wavelengths," Astrophys. J. (Lett.) 558, p. 65, 2001.

30. D. Maia, M. Pick, A. Vourlidas, and R. Howard, "Development of Coronal Mass Ejections: Radio Shock Signatures," Astrophys. J. (Lett.) 528, pp. L49-L51, 2000.

31. H. V. Cane, W. C. Erickson, and N. P. Prestage, "Solar flares, type iii radio bursts, coronal mass ejections and energetic particles," J. Geophys. Res. , 2002. in press.

32. J. C. James, "Radar Studies of the Sun," in Radar Astronomy, pp. 323-385, McGraw Hill, 1968.

33. W. A. Coles, "Prospects for a Solar Radar at Arecibo," 200th American Astronomical Society Meeting, Albuquerque 49.09, p. 722, 2002.

34. B. Thidé and the LOIS Science Team, "Radio studies of solar terrestrial relationships," LOFAR Science Memo 5 , 2002. 